\title{
Inspection of Delamination Defect in First Wall Panel of Tokamak Device by Using Laser Infrared Thermography Technique
}

\author{
Haochen Liu ${ }^{(}$, Cuixiang Pei ${ }^{\circledR}$, Jinxing Qiu, and Zhenmao Chen
}

\begin{abstract}
First wall panels (FWPs), which adjoin the inner wall of the blanket modules in the vacuum vessel (VV) of a Tokamak device, are in structures of multilayer bounded together with a solid welding technique in order to perform its heat exchange, VV protection, and neutron breeding functions. The quality of the welding joint between layers is the key factor for FWP integrity. In order to conduct online inspection of the delamination defect in the FWPs, a nondestructive testing (NDT) method capable to detect delamination defect without accessing into the $\mathrm{VV}$ is required. In this paper, the feasibility of the laser infrared thermography (LIRT) testing NDT method was investigated experimentally for this purpose. To clarify its detectability under practical VV environment, inspections of several inspection modes were conducted based on the practical structure of FWP and VV of the EAST Tokamak device, i.e., modes of different distances and angles of FWPs toward the LIRT transducers. In practice, an LIRT testing system was established and several double-layered plate specimens with different artificial delamination defects were inspected under the selected testing conditions. Through thermography signal reconstruction, an image processing algorithm was proposed and adopted to enhance the defect detectability. From the results of different inspection modes, it was found that the angle factor may worsen the inspection precision and reduce the detectability for delamination defects in case of big defect depth-to-width ratio, even though the LIRT method is still applicable for inspection of relative large defects in FWP. Finally, the detectability in different inspection modes was clarified, which proved the feasibility of LIRT for FWP online inspection.
\end{abstract}

Index Terms-Blankets, first wall panel (FWP), image enhancement, infrared thermography (IRT), online inspection, Tokamak.

\section{INTRODUCTION}

$\mathbf{I}^{2}$ $\mathrm{N}$ TOKAMAK fusion nuclear device, multilayered plate is a typical mechanical structure similar to that in blanket modules and divertors, which are two major components in the vacuum vessel (VV). The first wall panel (FWP)

Manuscript received June 30, 2017; revised September 26, 2017; accepted January 5, 2018. Date of publication April 16, 2018; date of current version July 9, 2018. This work was supported in part by the National Magnetic Confinement Fusion Program of China under Grant 2013 GB113005 and in part by the National Natural Science Foundation of China under Grant 51577139 and Grant 51407132. The review of this paper was arranged by Senior Editor E. Surrey. (Corresponding author: Zhenmao Chen.)

The authors are with the State Key Laboratory for Strength and Vibration of Mechanical Structures, Xi'an Jiaotong University, Xi'an 710049, China (e-mail: 1hc0608@stu.xjtu.edu.cn; pei.cx@mail.xjtu.edu.cn; qjxsang@stu.xjtu.edu.cn; chenzm@mail.xjtu.edu.cn).

Color versions of one or more of the figures in this paper are available online at http://ieeexplore.ieee.org.

Digital Object Identifier 10.1109/TPS.2018.2803282 of blanket module consists of multilayered plate of three metallic materials [beryllium, copper, and reduce activiation ferritic/martensitic (RAFM) steel] bonded together using the hot isostatic pressure welding technique. One of the major concerns of the FWP is the delamination defect at the interface between layers. Such defect may seriously worsen heat transfer and causes thermal stress problem [1]-[3]. Therefore, the nondestructive testing (NDT) of delamination defect in FWP is of great importance. However, it is difficult to apply the conventional NDT techniques, such as the ultrasonic testing and the eddy current testing, to detect the delamination of FWP due to the special environment in the VV, especially for an online case. Infrared thermography (IRT) testing technique was proposed recently for nondestructive evaluation of such structures [4].

The IRT method has drawn a lot of attention because of its features of remote sensing, noncontacting, and high detection efficiency [5]-[7]. Several studies of the IRT inspection of the plasma facing components have been reported recently [8]-[11]. They mainly focused on the inspection of monoblocks in the divertor during their fabrication procedure. The feasibility of the IRT method for the FWP online inspection is not yet clear. Concerning the features of the structure and environment of Tokamak, the online inspection of FWP has to be performed outside the VV through its windows. For this reason, the laser IRT (LIRT) testing method, which is featured with long-distance inspection, good directionality, and high inspection efficiency, is a good candidate to do the online inspection of the FWPs in a practical Tokamak device.

However, it is well known that the best angle of the LIRT measurement is $90^{\circ}$, i.e., inspection perpendicular to the FWP. Moreover, a longer distance between the transducer and the objects may also reduce the signal-to-noise ratio in the LIRT inspection. Therefore, to apply the LIRT for inspection of delamination in the FWPs, to clarify the influence of the practical environment of a Tokamak device to the LIRT method is of great importance, which is the aim of this paper.

The contents of this paper are arranged as follows. First, a high-efficiency numerical simulation method of IRT developed by authors is briefly introduced. Based on the structure of the EAST Tokamak, several typical inspection modes, which mainly focus on the features of distance and angles between the inspection transducers and the objects, are selected. Second, the feasibility of the LIRT method for delamination inspection in multilayered FWP is validated through both 
numerical simulation and experiments. Third, a signal processing algorithm is described for the boundary extraction of the delamination defects. Finally, the detectability under practical environment is evaluated based on a series of experimental results under selected inspection modes.

\section{Numerical Simulation Method AND INSPECTION MODES}

\section{A. Numerical Simulation Method}

To evaluate the feasibility of delamination defect inspection using the LIRT technique numerically, a numerical simulation code developed by the authors is applicable [12]. The basic principle of the method is as follows.

The finite element method (FEM) governing equation of the LIRT problem can be written as

$$
[K]\{T(t)\}+[C]\left\{\frac{\partial T(t)}{\partial t}\right\}=\{Q(t)\}
$$

where $\{Q(t)\}$ denotes the excitation heat source and $[\kappa],[C]$ are the coefficient matrices.

Since the LIRT problem is a linear one fulfilling the principle of superposition, the response signal of the pulsed LIRT can be calculated with the frequency-domain summation method. In practice, using the Fourier transformation and the complex approximation, the governing equation (1) can be rewritten as

$$
\sum_{n=0}^{N}\left([\kappa]+j \omega_{n}[C]\right)\left\{\tilde{T}_{n}\right\} e^{j \omega_{n} t}=\sum_{n=0}^{N}\left\{\tilde{Q}_{n}\right\} e^{j \omega_{n} t}
$$

where $\tilde{T}_{n}$ and $\tilde{Q}_{n}$ are the amplitude of the $n$th harmonic component, $\omega_{n}$ is the angular frequency of the $n$th sinusoidal harmonic component, and $N$ is the total number of the harmonic components that is selected according to the accuracy requirement. From (2), the following equations can be derived as:

$$
\left([\kappa]+j \omega_{n}[C]\right)\left\{\tilde{T}_{n}\right\}=\left\{\tilde{Q}_{n}\right\}, \quad n=0,1,2, \ldots \ldots, .
$$

From (3), the response of each frequency component can be solved, and the pulsed IRT signals can be finally obtained by summing up all the components. To guarantee the simulation precision and enhance the simulation efficiency, two strategies are proposed [10]. First, the number of necessary harmonic frequency components $N$ can be reduced using an interpolation strategy. Second, a fast forward scheme based on the databases strategy is the applicable calculation of the temperature perturbation of each frequency component.

The numerical model of the IRT problem for multilayer plate is shown in Fig. 1, where $\Omega$ denotes the whole no-defect object, $\Omega_{c}$ is the region of delamination, and $\Omega_{0}$ denotes the delamination region. The fast forward scheme based on the databases strategy can be described as follows based on this numerical model.

Upon subtracting the single frequency IRT governing equation about $T^{0}$, temperature of the unflawed model, from that about $T$, the temperature of the model with defect, one can obtain the governing equations about the field perturbation for single frequency IRT problem. After separately denoting all

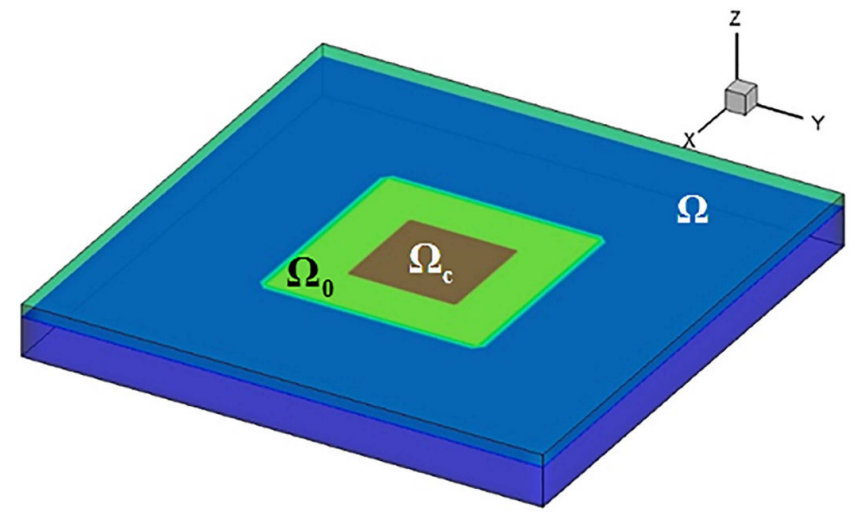

Fig. 1. Numerical model of the fast numerical simulation method.

the nodes as three parts, the FEM equations about $T^{f}$ of the IRT problem become

$$
\left[\begin{array}{ccc}
\bar{K}_{11} & \bar{K}_{12} & \bar{K}_{13} \\
\bar{K}_{21} & \bar{K}_{22} & \bar{K}_{23} \\
\bar{K}_{31} & \bar{K}_{32} & \bar{K}_{33}
\end{array}\right]\left\{\begin{array}{l}
T_{1}^{f} \\
T_{2}^{f} \\
T_{3}^{f}
\end{array}\right\}=\left[\begin{array}{ccc}
\tilde{K}_{11} & 0 & 0 \\
0 & 0 & 0 \\
0 & 0 & 0
\end{array}\right]\left\{\begin{array}{l}
T_{1}^{f}+T_{1}^{0} \\
T_{2}^{f}+T_{2}^{0} \\
T_{3}^{f}+T_{3}^{0}
\end{array}\right\}
$$

where $[\bar{K}]$ is the FEM coefficient matrix of the unflawed model, $[\tilde{K}]$ is the coefficient matrix corresponding to the model with defect, $\left\{T_{1}\right\}$ is the temperature at the nodes of delamination elements, $\left\{T_{3}\right\}$ is the temperature at the inspection surface nodes, and $\left\{T_{2}\right\}$ is the remained unknowns. By denoting the inverse matrix of $[\bar{K}]$ as $[H]$, the following equations correlating the unflawed field to the temperature perturbation due to defect can be derived as:

$$
\begin{aligned}
{\left[I-H_{11} \tilde{K}_{11}\right]\left\{T_{1}^{f}\right\} } & =\left[H_{11}\right]\left[\tilde{K}_{11}\right]\left\{T_{1}^{0}\right\} \\
\left\{T_{3}^{f}\right\} & =\left[H_{31} \tilde{K}_{11}\right]\left\{T_{1}^{f}+T_{1}^{0}\right\} .
\end{aligned}
$$

Since the coefficient submatrices $\left[H_{11}\right], \quad\left[H_{31}\right]$ and the unflawed temperature vectors $\left\{T_{1}^{0}\right\},\left\{T_{3}^{0}\right\}$ are independent of the defect geometry, once they have been calculated a priori and stored as databases, there is no need to compute them again for defects of a different profile. Thus, the fast forward scheme can remarkably promote the simulation efficiency as the dimension of (5) is much smaller than full system [13].

\section{B. Inspection Modes Analysis}

To implement the LIRT method for the inspection of FWPs through the window of $\mathrm{VV}$, it is important to investigate the influence of the practical inspection conditions. The distance and angles between the target FWP and the inspection system are the key factors of the practical online inspection of FWPs.

The EAST Tokamak, which is the first full superconducting Tokamak with noncircle cross section of VV and actively cooled plasma facing components, has been built in the Institute of Plasma Physics, Chinese Academy of Sciences. The actual parameters of VV in the EAST are adopted to extract the key environmental feature parameters for LIRT inspection.

Fig. 2 shows the top-view blueprint of VV of EAST. The inspection system is set outside one of the flange windows. 


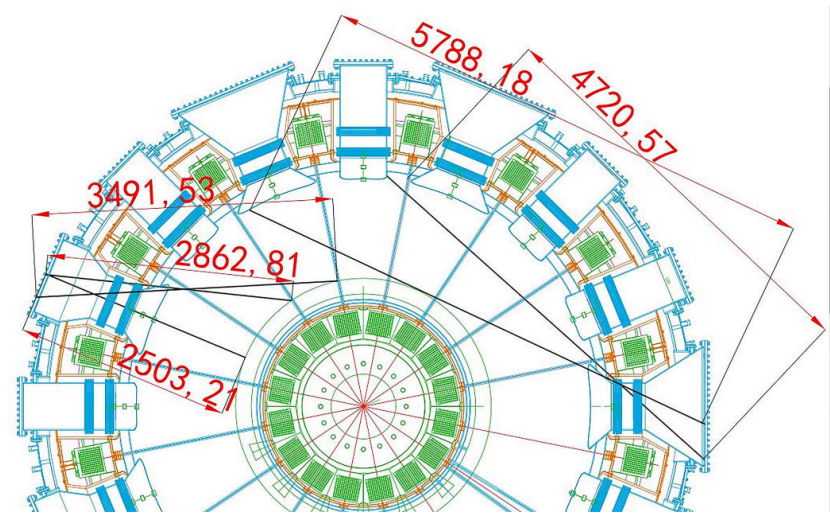

Fig. 2. Top view blueprint of VV.

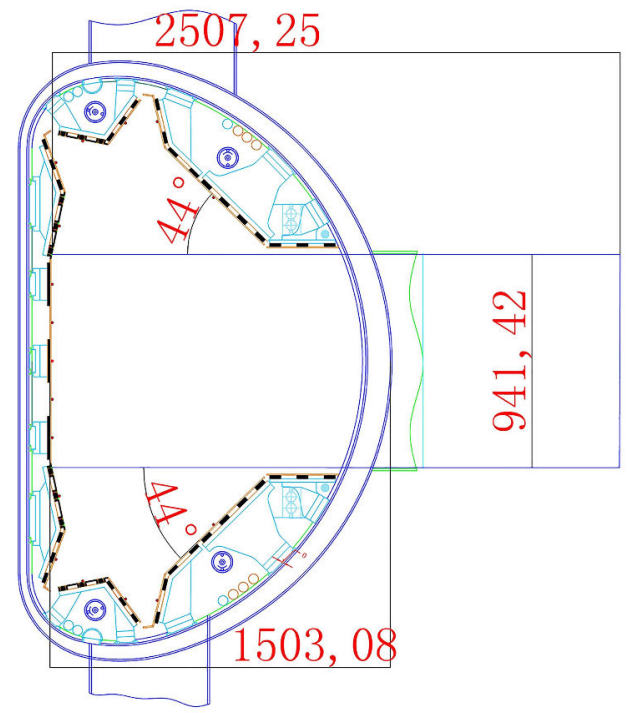

Fig. 3. Side view blueprint of one part of VV.

The distances and angles can be extracted based on the practical structure and size. As for LIRT measurements shown in Fig. 2, the maximum distance of the blankets on the outer side to the inspection window is over $5.0 \mathrm{~m}$. The minimum angle of blankets toward the IR camera is $35^{\circ}$. Therefore, from a given window, FWPs on three inner sides and two outer sides blanket modules can be detected physically. In addition, as presented in Fig. 3, which is the side view blueprint of one part of the $\mathrm{VV}$, not only horizontal angles but also a $44^{\circ}$ vertical angle of FWP should be considered for inspection of the outer side blankets. Therefore, five inspection modes (three inner and two outer cases) as listed in Table I are necessary to be considered.

From the point of view of practical inspection, the influence of inspection angle is more significance than that of the distance, because the influences caused by the distance variation can be eliminated by changing camera lens and shaping laser spots of inspection system. Therefore, three inspection modes
TABLE I

INSPECTION MODES

\begin{tabular}{lc}
\hline \hline \multicolumn{1}{c}{ Item } & Details of distance and angle features \\
\hline $\begin{array}{l}\text { Detectable FW blanket } \\
\text { modes }\end{array}$ & $\begin{array}{l}\text { Inner: } 2.5 \mathrm{~m} / 90^{\circ} ; 2.8 \mathrm{~m} / 65^{\circ} ; 3.4 \mathrm{~m} / 35^{\circ} ; \\
\text { Outer: } 4.7 \mathrm{~m} / 60^{\circ}-44^{\circ} ; 5.8 \mathrm{~m} / 35^{\circ}-44^{\circ} ;\end{array}$ \\
$\begin{array}{l}\text { Inspection modes in lab } \\
\text { experiments }\end{array}$ & $1.8 \mathrm{~m} / 90^{\circ} ; 2.2 \mathrm{~m} / 60^{\circ}-44^{\circ} ; 2.5 \mathrm{~m} / 35^{\circ}-44^{\circ}$ \\
\hline \hline $\begin{array}{l}\text { The details of distance and angle of mode is marked in form as } \\
\text { "Distance / Angle (horizontal) - Angle (vertical)" }\end{array}$
\end{tabular}

of different FWP angles shown in Table I are selected as major inspection conditions to be evaluated.

\section{LIRT Method Powered By TSR Processing}

The LIRT generates a shaped laser spot, which can concentrate the heat power to the target sample and guarantee a uniform heating. Only a single pulse is required in this method, which can significantly reduce the imaging time. The thermographic signal reconstruction (TSR) method is also applicable to enhance the inspected image of the LIRT for a higher defect contrast [14].

TSR is an effective method to process the transient thermal images of pulse thermography experiments [15]. The basic algorithm of TSR is as that shown in Fig. 4. It can effectively prevent noise interference along the transient temperature curve. It has been proven that the logarithmic behavior of temperature decay exhibits remarkable consistency in the pixels at defect free area, and those corresponding to subsurface defect departing from the near-linear signature at a particular time instant. This phenomenon is important to distinguish between intact and defect area. In practice, it is rational to approximate the logarithmic curve of temperature-time during decay by polynomial fitting on each pixel as given in

$$
\begin{aligned}
\log _{10}(\Delta T(t))=a_{0}+ & a_{1} \log _{10}(t) \\
& +a_{2}\left[\log _{10}(t)\right]^{2}+a_{n}\left[\log _{10}(t)\right]^{n}
\end{aligned}
$$

where $a_{n}$ is the $n$th order polynomial coefficient.

Typically, order $n$ polynomial function can be set to 5 or 6 to avoid the high-order noise and ensure a good correspondence between the actual data and the fitting values. Based on these considerations, the first and second logarithmic derivatives of the thermograms can be calculated using

$$
\begin{aligned}
\frac{d\left(\log _{10}(\Delta T(t))\right)}{d\left(\log _{10}(t)\right)} & =\sum_{n=0}^{N} n a_{n} \log _{10}(t)^{n-1} \\
\frac{d^{2}\left(\log _{10}(\Delta T(t))\right)}{d\left(\log _{10}(t)\right)^{2}} & =\sum_{n=0}^{N} n(n-1) a_{n-1} \log _{10}(t)^{n-2} .
\end{aligned}
$$

In practice, the first logarithmic derivatives are selected and the sound area cases are also inspected as reference cases. The difference in the first logarithmic derivative image between the defect case and the reference sound case is calculated to enhance defect contrast. 


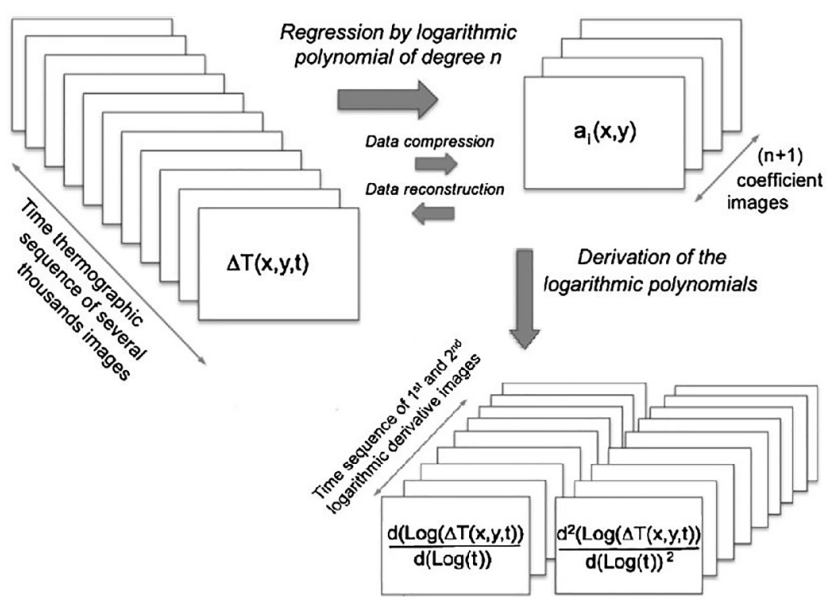

Fig. 4. Algorithm chart of the TSR method.

TABLE II

SPECIFICATIONS OF SPECIMENS

\begin{tabular}{|c|c|}
\hline $\begin{array}{l}\text { Double-layered } \\
\text { Specimen }\end{array}$ & Sizes and materials \\
\hline Al-Cu specimen & $\begin{array}{l}\text { Rectangle plate with square delamination } \\
\text { defects } \\
\text { Height: } 100 \mathrm{~mm} \text {; Width: } 100 \mathrm{~mm} \text {; } \\
\text { Thickness: } 10.5 \mathrm{~mm} \text {; } \\
\text { Al layer Thickness: } 5.5 \mathrm{~mm} \text {; } \\
\text { Cu layer Thickness: } 5.0 \mathrm{~mm} \text {; }\end{array}$ \\
\hline SUS304 specimen & $\begin{array}{l}\text { Rectangle plate with circle and ellipse } \\
\text { delamination defects } \\
\text { Height: } 130 \mathrm{~mm} \text {; Width: } 130 \mathrm{~mm} \text {; } \\
\text { Thickness: } 8.3 \mathrm{~mm} \text {; } \\
\text { First SUS304 layer Thickness: } 3.3 \mathrm{~mm} \text {; } \\
\text { Second SUS304 layer Thickness: } 5.0 \mathrm{~mm} \text {; }\end{array}$ \\
\hline
\end{tabular}

Usually, the derivative images of the thermograms depend on the time instant, which lead to a possible selection of the best derivative images in the whole image sequence. The best image can be selected as the image when the first maximum difference value on the defect area happens along the images sequence. The so-obtained best images have much better defect contrast than the raw thermographic images.

\section{EXPERIMENTAL DETAILS}

\section{A. Specimen Preparation}

The practical FWP in Tokamak is a multilayered structure with different materials, such as beryllium, copper alloy, and RAFM/stainless steel in International Thermonuclear Experimental Reactor. As the most delamination defects happen at the bonding interface between the first two layers, simulated specimens were designed and manufactured as double-layered plates, bonded together using the diffusion welding technique. Two double-layered plates, denoting as $\mathrm{Al}-\mathrm{Cu}$ specimen and SUS304 specimen, were fabricated with sizes and materials shown in Table II.

The delamination defects in the specimens were simulated as a square or elliptic thin volume at the bottom of upper layer. Fig. 5 shows the shape, size, and the distribution of delamination defects in the two specimens.

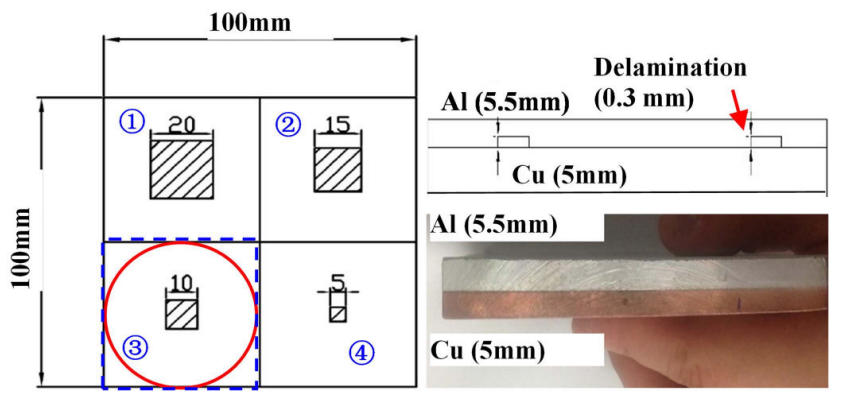

(a)

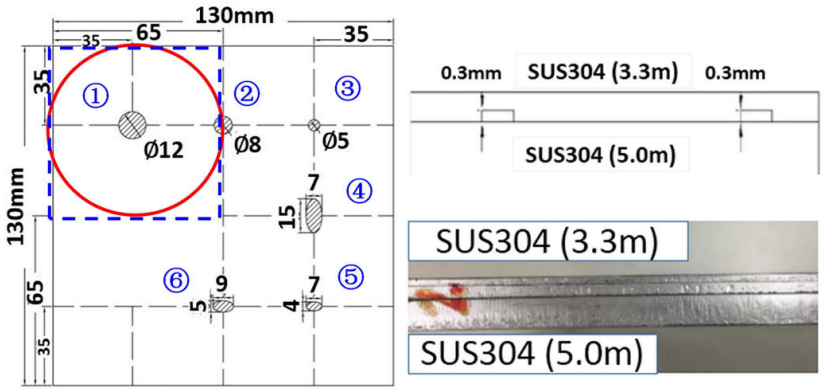

(b)

Fig. 5. Schematic of specimens. (a) Al-Cu double-layered specimen. (b) SUS304 double-layered specimen.

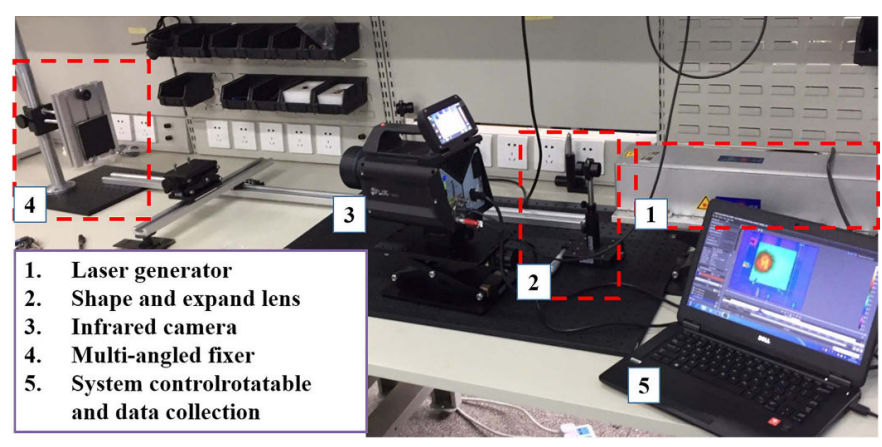

Fig. 6. Experimental system.

\section{B. Experimental System}

In order to implement the inspection of the selected modes, an LIRT experimental system was established. The experimental setup is shown in Fig. 6. The thermal images were acquired by an FLIR-X6530sc mid-wave infrared thermal camera, with a spectral range of 3.7-5.0 $\mu \mathrm{m}$ and an indium antimonite detector. The resolution of recorded images is $640 \times 512$ pixels. The camera was connected and controlled by a PC. The exciting laser was generated and shaped by a set of optical lens to reach a relatively homogenous circle region of laser spot. A function generator provides a square pulse wave to the laser generator module to supply a $100-\mathrm{W}$ square pulsed laser. In this way, the uniform laser spot was generated to heat the specimen.

The specimen was dark painted and installed on a special designed rotatable fixture to implement different angles toward the camera and laser beam. The rotatable fixer can adjust not only the distances between specimen and camera, but also the horizontal and vertical angles. Attributed to the shape lens, 


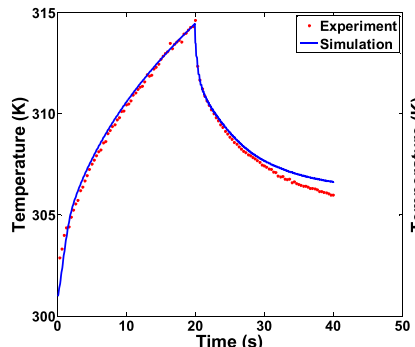

(a)

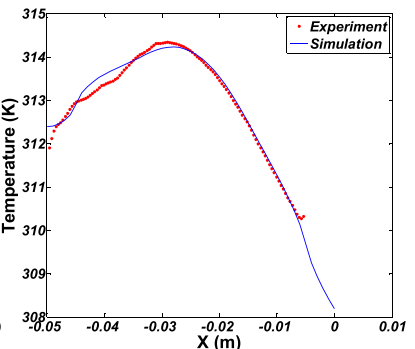

(b)
Fig. 7. Comparison results between simulation and experiment. (a) Transient temperature of center point. (b) Temperature of center line at the time of pulse end.

the laser spot was controlled to focus on the specimen surface to get better heating efficiency. At last, the whole experimental system was sealed into a darkroom to isolate the noise of outside light.

The laser induced on the specimen is a 20-s square pulse. During heating, thermal images were captured at the end of the laser pulse by camera with a $60-\mathrm{Hz}$ frame rate. A total of 1200 images were taken and recorded to ensure enough transient decay temperature signals.

In experiments, the laser spot was focused on a small heating area (represented by the solid circle in Fig. 5), corresponding to each defect, respectively, because the maximum laser power cannot guarantee high enough heat flux to heating the whole specimen surface. Therefore, the area surrounded by a rectangular dashed line in Fig. 5 is the region of interest (ROI) corresponding to each defect. In addition, as the laser path is fixed in our system, the switching heating location is realized by moving the specimen instead of adjusting laser path. Therefore, though it seems that the heating area is the same in different images of the following experimental results, the corresponding defect was different.

The sound reference experiments of the $\mathrm{Al}-\mathrm{Cu}$ and the SUS304 specimen are carried out in the same experimental conditions. Instead of inspecting an unflawed specimen, the sound cases for the Al-Cu specimen is located at the center of the $\mathrm{Al}-\mathrm{Cu}$ plate. For SUS304 specimen, it is located at a spare area without defects embedded.

\section{NUMERICAL AND EXPERIMENTAL RESULTS}

\section{A. Numerical Simulation}

A double-layered plate model was simulated to investigate the feasibility of delamination inspection by the LIRT techniques. The schematic of the double-layered model is shown in Fig. 1. The size, material of the model, and delamination defect distribution are consistence with those of the Al$\mathrm{Cu}$ specimen, which are shown in Fig. 5(a). The square delamination defect of $20-\mathrm{mm}$ side length was considered.

Fig. 7 shows the comparison between the simulation result and the inspected raw thermal image. The transient temperature of the center point and centerline temperature at the pulse ending time are compared in the figure. The location of the center point and centerline are marked as the dashed line in the upper left raw of the thermal image in Fig. 9(a).



Fig. 8. ROI of the best images of simulation results.

From the numerical results, it is clear that the simulation method can precisely predict the temperatures on many feature signals, including peak value, rising and falling edge, and temperature distribution around the surface below the defects. Though a relative big error may happens at the steady state, it is fortunately not important for practical inspection. The good agreement between numerical and experimental results at selected time instant demonstrated the validity of the proposed numerical method and the code. Based on the simulation results, the best first logarithmic derivative image is also calculated and presented in Fig. 8, which clearly indicates a higher defect contrast. The simulation results demonstrated the feasibility of the delamination defect using the LIRT technique.

\section{B. Calibrations}

At first, the inspection of $1.8-\mathrm{m}$ distance and $90^{\circ}$ angle mode was implemented and a calibration was carried out based on the results of this direct facing mode.

The calibration samples were used to demonstrate the detectability of the developed experimental system for the delamination defects and the efficiency of the image processing algorithm. The inspection experiments of the two relative large defects of each specimen were selected for calibration. In the $\mathrm{Al}-\mathrm{Cu}$ specimen, the calibration cases are ones of square $20 \mathrm{~mm}$ and square $15-\mathrm{mm}$ defects. Likewise, the $12-\mathrm{mm}$ diameter circle defect and $15 \times 7 \mathrm{~mm}^{2}$ elliptical defects were selected as the calibration cases for the SUS304 specimen. The roughness of the specimen surface also provides a realistic level of noise to the measurements.

The raw thermal images of calibration cases and sound reference cases measured at the terminating time of laser pulse are shown in Fig. 9. The raw thermograms indicate the real sight of camera sampling and temperature distributions at the surface of the tested specimens. The centerline temperature of calibration cases and sound reference cases extracted from the thermal images are presented in Fig. 10, where the noise level and raw temperature signals are illustrated. It clearly informs that the sensitivity of the measurement systems is satisfactory for practical inspection.

As expected, the defects are not clearly visible in the raw thermal images. The ROIs' references to each defect, which are shown as black rectangle area in Fig. 9, were extracted from each raw image to enhance image process efficiency. The first logarithmic derivative images of the TSR method 


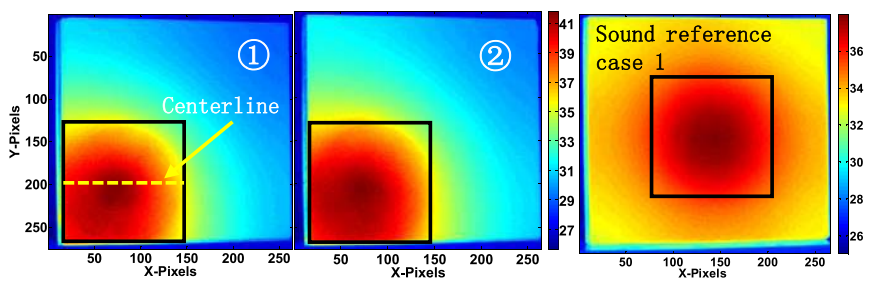

(a)

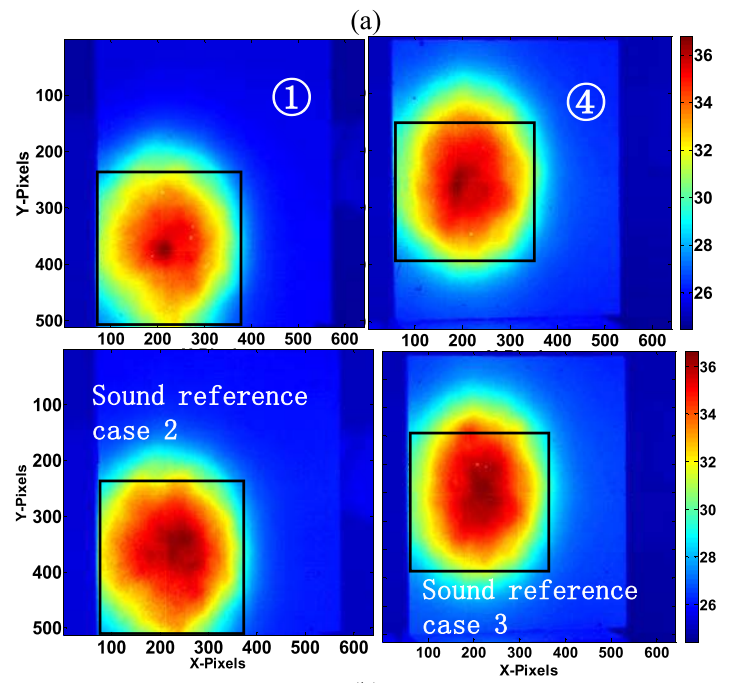

(b)

Fig. 9. Raw thermal images of calibration cases and sound reference cases. (a) 20-mm and 15-mm defect in Al-Cu specimen and sound reference case. (b) $\Phi$ 12-mm circle and 15-7-mm ellipse defect in SUS304 specimen and sound reference cases.

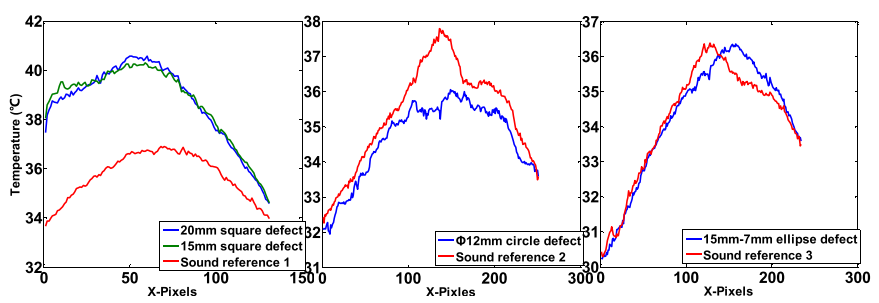

Fig. 10. Comparison of the temperature of the centerline between the calibration cases and the sound reference cases.

were then calculated from the thermal data. For one certain depth delamination defects, the best contrast images are at the same place in the sequence of first derivative images. The best first logarithmic derivative images of defects in the Al-Cu and the SUS304 are at the time 0.5 and $2.1 \mathrm{~s}$, respectively.

The difference of the best images between calibration and sound reference cases was calculated and shown in Fig. 11, which reveals a significantly enhanced defect contrast. After processing, the defects become clearly visible. The difference values of centerline of four calibrations are presented in Fig. 12, which indicate that the size and boundary of defects cannot be defined by only using the difference values.

For further defect contrast enhancement, based on threshold concept, a sizing algorithm was applied to identify the boundary of the defects using the actual calibration data fork [16]. The goals were both to calculate an appropriate threshold value and to determine if a percentage threshold is applicable for defects of different sizes at different levels of thermal contrast.

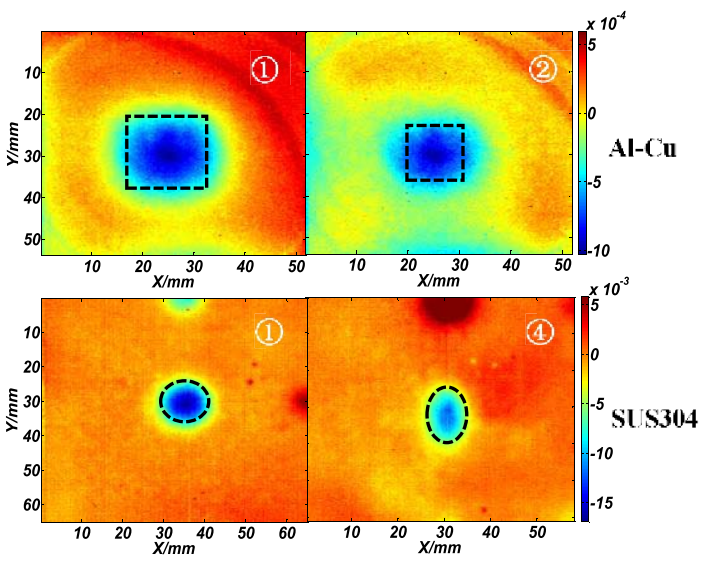

Fig. 11. Best difference derivative images of calibration cases.

TABLE III

DIFFERENCE DERIVATIVE STATISTICS FOR EACH DEFECT IN CALIBRATION

\begin{tabular}{ccccc}
\hline \hline \multirow{2}{*}{ Parameter } & \multicolumn{2}{c}{$\mathrm{Al}+\mathrm{Cu}$} & \multicolumn{2}{c}{ SUS304 } \\
& $20 \mathrm{~mm}$ & $15 \mathrm{~mm}$ & $\mathrm{Circle} \varnothing 12$ & Eclipse(7,15) \\
\hline $\begin{array}{c}\text { Maximum } \\
\text { value }\end{array}$ & -0.00161 & -0.00107 & -0.0199 & -0.0146 \\
$\begin{array}{c}\text { Average } \\
\text { value }\end{array}$ & -0.00098 & -0.00062 & -0.0111 & -0.0083 \\
Threshold & $61.1 \%$ & $60.17 \%$ & $56.2 \%$ & $57 \%$ \\
\hline \hline
\end{tabular}

First, calculations of actual boundary region for four calibration defects were carried out for images shown in Fig. 11 using the specimen of size shown in Table II to obtain the corresponding dimensions of each pixel. The location of each defect was then mapped onto images in Fig. 11. The maximum difference derivative value was calculated within each boundary, and the average value of all pixels in the boundary was also calculated. The data for each defect are shown in Table III, where the threshold was defined as the ratio of the average value to the maximum value.

Based on the results shown in Table III, $61 \%$ and 57\% boundary extraction threshold were defined for the two specimens, respectively. The two threshold criterions were then applied and mapped to the pixels in the best images of all the defects of the two specimens to obtain the threshold binary images. Based on the threshold binary images, the defect edge profiles were extracted for as the final results.

The defect boundary results obtained with the strategy described earlier are shown in Fig. 13. All defects in two specimens are clearly detected. Therefore, in the case of the $1.8-\mathrm{m} / 90^{\circ}$ mode, the detectability for the $\mathrm{Al}-\mathrm{Cu}$ specimen can reach to defect as small as $5-\mathrm{mm}$ side length, which means 1.0 depth-to-width ratio.

With the decrement in the defect size, the extracted boundary becomes blurry and the noise in sound area starts showing up. Compared with the SUS304 specimen, there are two reasons that account for more sharp defects contrast and pure image in the SUS304 results. One is that the higher thermal 


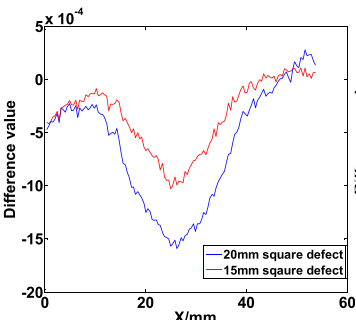

(a)



(b)
Fig. 12. Difference value of centerline of calibration case. (a) Two calibration defects in Al-Cu specimen. (b) Two calibration defects in SUS304 specimen.

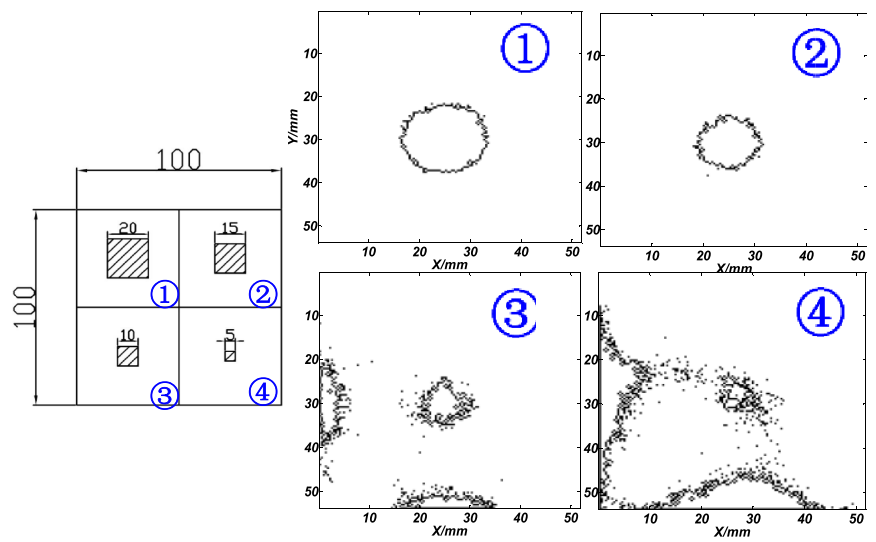

(a)

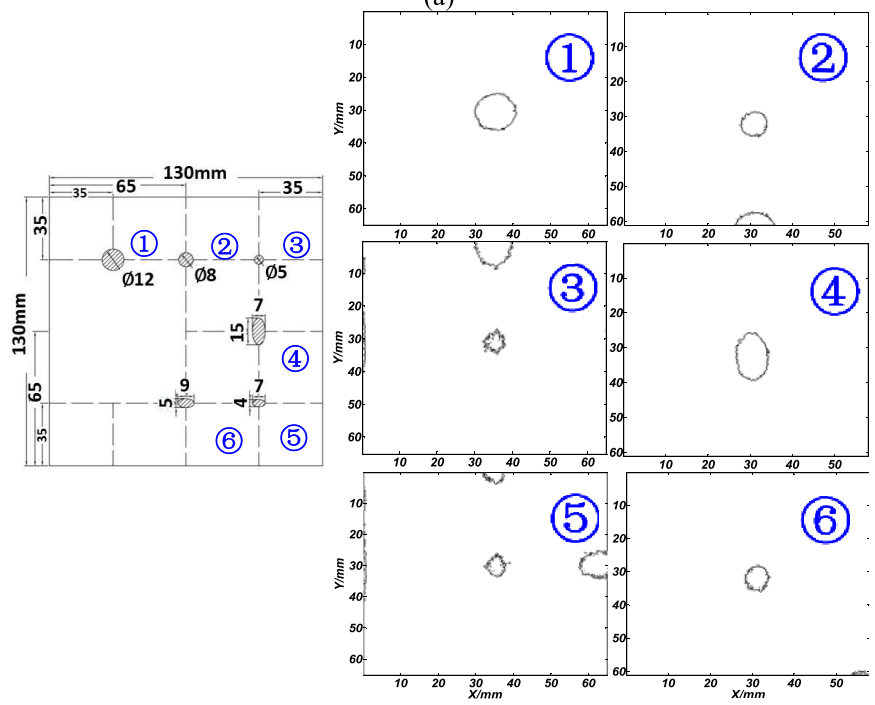

(b)

Fig. 13. Defect boundary results. (a) Al-Cu specimen. (b) SUS304 specimen.

conductivity of $\mathrm{Al}$ and $\mathrm{Cu}$ and deeper defects. The other is that the location of sound reference case of the $\mathrm{Al}-\mathrm{Cu}$ specimen is not fully correspondent to the defect locations. The boundary effect brings bigger noise and causes impurity in the results of the small defects in the $\mathrm{Al}-\mathrm{Cu}$ specimen.

\section{Angle Modes Inspection}

As discussed in Section II, the other two inspection modes (distance change and angles rotation) were also conducted with the same experimental system. The analysis of



Fig. 14. Defect boundary results of $\mathrm{Al}-\mathrm{Cu}$ in $2.2 \mathrm{~m}-60^{\circ}-44^{\circ}$ modes.
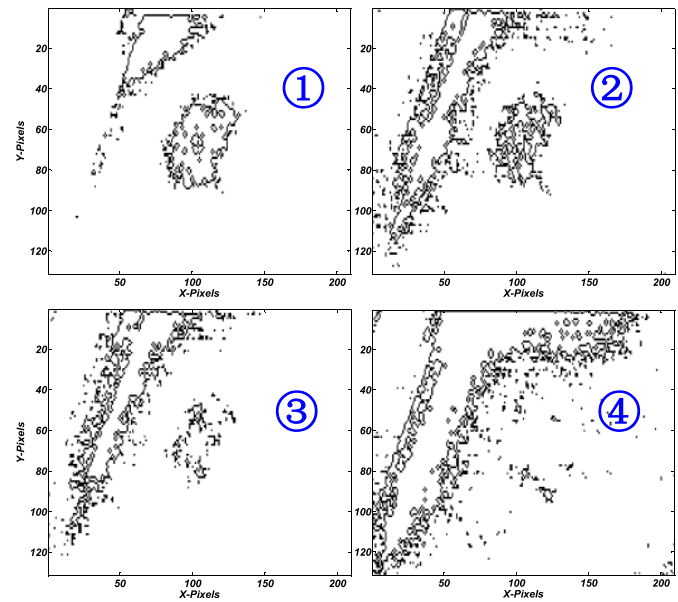

Fig. 15. Defect boundary results of $\mathrm{Al}-\mathrm{Cu}$ in $2.2 \mathrm{~m}-35^{\circ}-44^{\circ}$ modes.

these images was driven by the results from the calibration specimen given in Section V-B.

The thermal images were processed by using the TSR method and sizing algorithm. In this case, when applying the threshold criterion obtained from Section V-B, the ROIs contain the side surface of specimen, which confused the maximum value selection for threshold factor. As this research is mainly concerned about the detectability investigation, the selection of the maximum value was then carried out in the area on the top surface and intentionally avoided the pixels that can be easily recognized as surface noise. In this way, the defect boundaries were obtained.

Figs. 14 and 15 show the results of the $\mathrm{Al}-\mathrm{Cu}$ specimen in the $2.2 \mathrm{~m} / 60^{\circ}-44^{\circ}$ and $2.2 \mathrm{~m} / 35^{\circ}-44^{\circ}$ modes. The results of the SUS304 specimen are presented in Figs. 16 and 17. It is not difficult to find that defects boundary were transformed and even more blurry with an increasing specimen rotation angle. This is because the infrared emissivity mainly focuses on the normal direction of surface, even though, all defects in the SUS304 specimen are still clearly visible with rough boundary. However, the detectability limit for the $\mathrm{Al}-\mathrm{Cu}$ specimen was reduced in the angle modes results. The 5-mm 




Fig. 16. Defect boundary results of SUS304 in $2.2 \mathrm{~m}-60^{\circ}-44^{\circ}$ modes.

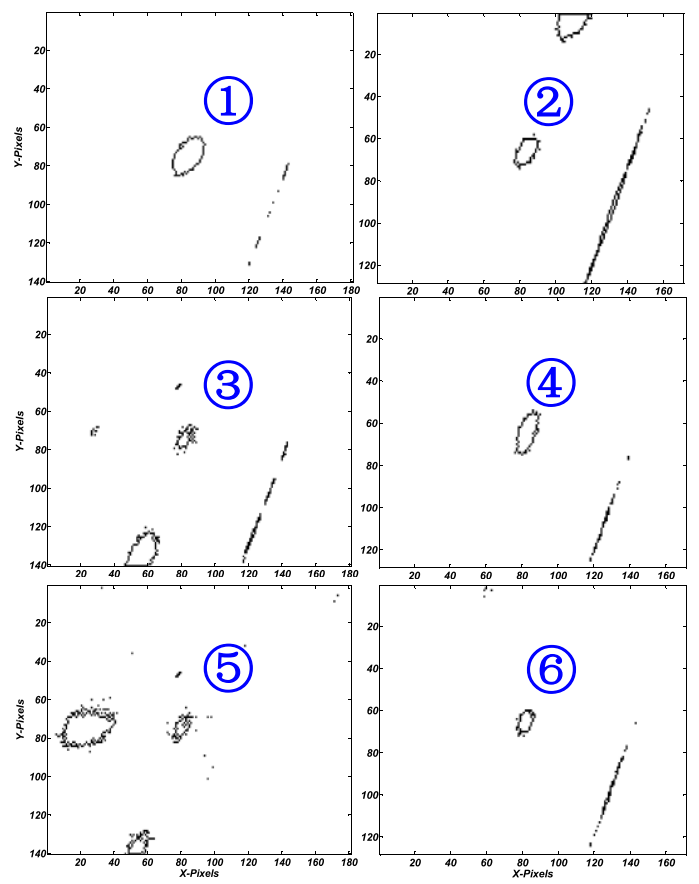

Fig. 17. Defect boundary results of SUS304 in $2.2 \mathrm{~m}-35^{\circ}-44^{\circ}$ modes.

square delamination in the $\mathrm{Al}-\mathrm{Cu}$ specimen is difficult to be recognized in angle modes. The main reason is that the bigger noise confused the selection of the maximum value for small defect. The contrast enhancement due to the threshold factor has been significantly restricted in this case.

\section{CONCLUSION}

In this paper, the feasibility of the LIRT technique for online detection of the delamination defect in FW blankets was investigated. With numerical and experimental investigation, the LIRT method was proven a promising NDT method for inspection of the delamination defect in FWPs. Considering the practical environment of FWPs in VV, detectability at typical inspection modes of different inspection distances and angles were investigated based on the structure of EAST Tokamak. Combining with the TSR method, a threshold-based sizing algorithm was implemented to provide an explicit defect contrast enhancement. Experimental results show that all the defects in the two specimens can be clearly detected using the LIRT images and the TSR algorithm in the direct facing mode. The detectability can reach to a depth-to-width ratio as small as 1.0. In angle mode cases, the detectability of the SUS304 specimen has not been significantly affected. However, the image of the smallest defect in the $\mathrm{Al}-\mathrm{Cu}$ specimen was submerged by noise, even though the defect of 10-mm side length can be clearly recognized in the angle mode. These experimental results reveal that the LIRT is feasible for the online inspection of FWPs of the blankets in VV of EAST Tokamak.

In the future research, apart from delamination defects, it is necessary to inspect the dry joint defects, which also happens in welding interface. Though the thermal conductivity of real dry joint is much higher than that of delamination defect, it is still significantly different from that of the base material. Therefore, principally, the laser IR method can still be applied, but the detectability of small size dry joint may not as high as the delamination. This will be considered and investigated in the following research.

\section{REFERENCES}

[1] S.-K. Kim, E. H. Lee, J.-S. Yoon, H.-K. Jung, D. W. Lee, and B.-Y. Kim, "Preliminary test and evaluation of nondestructive examination for ITER first wall development in Korea," IEEE Trans. Plasma Sci., vol. 40, no. 9, pp. 2285-2289, Sep. 2012.

[2] T. Wang, "A continuum damage model for ductile fracture of weld heat affected zone," Eng. Fracture Mech., vol. 40, no. 6, pp. 1075-1082, 1991.

[3] T. J. Wang, "Unified CDM model and local criterion for ductile fractureI. Unified CDM model for ductile fracture,"Eng. Fracture Mech., vol. 42, no. 1, pp. 177-183, 1992.

[4] M. Aumeunier et al., "Simulation of the infrared views of the upper port VIS/IR imaging system of ITER," IEEE Trans. Plasma Sci., vol. 40, no. 3, pp. 753-760, Mar. 2012.

[5] J. Tashan and R. Al-Mahaidi, "Bond defect detection using PTT IRT in concrete structures strengthened with different CFRP systems," Composite Struct., vol. 111, pp. 13-19, May 2014.

[6] H.-C. Fernandes and X. Maldague, "Fiber orientation assessment in complex shaped parts reinforced with carbon fiber using infrared thermography," Quant. Infr. Thermograp. J., vol. 12, pp. 64-79, Apr. 2015.

[7] Y. He and R. Yang, "Eddy current volume heating thermography and phase analysis for imaging characterization of interface delamination in CFRP," IEEE Trans. Ind. Informat., vol. 11, no. 6, pp. 1287-1297, Dec. 2015.

[8] K. Kim et al., "Manufacturing and high heat flux testing of tungstenbrazed mock-ups for KSTAR divertor," IEEE Trans. Plasma Sci., vol. 45, no. 3, pp. 519-522, Mar. 2017.

[9] A. Durocher, N. Vignal, F. Escourbiac, J. L. Farjon, J. Schlosser, and F. Cismondi, "Development of an original active thermography method adapted to ITER plasma facing components control," Fusion Eng. Des., vols. 75-79, pp. 401-405, Nov. 2005.

[10] A. Durocher et al., "Infrared thermography inspection of the ITER vertical target qualification prototypes manufactured by European industry using SATIR," Fusion Eng. Des., vol. 84, pp. 314-318, 2009. 
[11] Y. Seki, K. Ezato, S. Suzuki, K. Yokoyama, M. Enoeda, and S. Mori, "Non-destructive examination with infrared thermography system for ITER divertor components," Fusion Eng. Des., vol. 85, nos. 7-9, pp. 1451-1454, 2010.

[12] H. Liu, S. Xie, C. Pei, and Z. Chen, "Numerical simulation method for IR thermography NDE of delamination defect in multilayered plate," Int. J. Appl. Electromagn. Mech., vol. 52, nos. 1-2, pp. 381-389, 2016.

[13] H. Liu, S. Xie, C. Pei, and Z. Chen, "Development of a fast numerical simulator for infrared thermography testing signals of delamination defect in a multilayered plate," IEEE Trans. Ind. Informat., to be published.

[14] D. L. Balageas, J.-M. Roche, F.-H. Leroy, W.-M. Liu, and A. M. Gorbach, "The thermographic signal reconstruction method: A powerful tool for the enhancement of transient thermographic images," Biocybern. Biomed. Eng., vol. 35, no. 1, pp. 1-9, 2015.

[15] J.-M. Roche, F.-H. Leroy, and D. Balageas, "Images of TSR coefficients: A simple way for a rapid and efficient detection of defects," Mater Eval, vol. 72, pp. 73-82, Jan. 2014.

[16] P. H. Shin, S. C. Webb, and K. J. Peters, "Pulsed phase thermography imaging of fatigue-loaded composite adhesively bonded joints," NDT \& E Int., vol. 79, pp. 7-16, Apr. 2016.

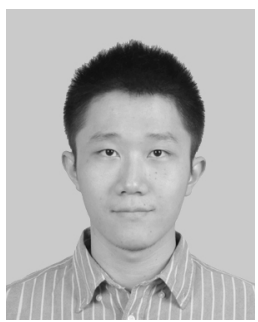

Haochen Liu is currently pursuing the Ph.D. degree with the School of Aerospace, Xi'an Jiaotong University, Xi' an, China.

His current research interests include the simulation and experiment and application of infrared thermography.



Cuixiang Pei received the Ph.D. degree from The University of Tokyo, Tokyo, Japan.

$\mathrm{He}$ is currently an Associate Professor with the Department of Engineering Mechanics, Xi'an Jiaotong University, Xi' an, China. His current research interests include the development and application of new NDT methods using EMAT, laser ultrasonic testing, and laser thermography testing.

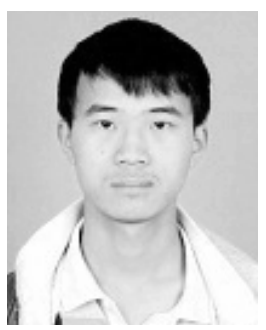

Jinxing Qiu is currently pursuing the Ph.D. degree with the School of Aerospace, Xi'an Jiaotong University, Xi'an, China.

His current research interests include the experiment and application of laser infrared thermography.



Zhenmao Chen received the Ph.D. degree from The University of Tokyo, Tokyo, Japan.

$\mathrm{He}$ is currently a Professor with the Department of Engineering Mechanics, Xi'an Jiaotong University, Xi' an, China. His current research interests include strength and vibration problems due to electromagnetic force, theory, and application of electromagnetic nondestructive testing (NDT) and inverse problems in NDT. 


\title{
Inspection of delamination defect in first wall panel of Tokamak device by using laser infrared thermography technique
}

\author{
Liu, Haochen
}

IEEE

Liu H, Pei C, Qiu J, Chen Z. (2019) Inspection of delamination defect in first wall panel of Tokamak device by using laser infrared thermography technique. IEEE Transactions on Plasma Science, Volume 46, Issue 7, July 2018, pp. 2699-2707

https://doi.org/10.1109/tps.2018.2803282

Downloaded from Cranfield Library Services E-Repository 